\title{
Study of the Double Nonlinear Quantum Resonances in Diatomic Molecules
}

\author{
Gustavo López, Jorge Gomez Tejeda Zanudo \\ Departamento de Física, Universidad de Guadalajara, Guadalajara, Mexico \\ E-mail:gulopez@udgserv.cencar.udg.mx,jgtz.fis@gmail.com \\ Received February 23, 2011; revised March 29, 2011; accepted April 19, 2011
}

\begin{abstract}
We study the quantum dynamics of diatomic molecule driven by a circularly polarized resonant electric field. We look for a quantum effect due to classical chaos appearing due to the overlapping of nonlinear resonances associated to the vibrational and rotational motion. We solve the Schrödinger equation associated with the wave function expanded in term of proper stationary states, $|n\rangle \otimes|\operatorname{lm}\rangle$ (vibrational $\otimes$ angular momentum states). Looking for quantum-classic correspondence, we consider the Liouville dynamics in the two dimensional phase space defined by the coherent-like state of vibrational states. We consider the relationship between the overlapping of the classical resonances and the mixing of the quantum states, and it is found some similarities when the quantum dynamics is pictured in terms of number and phase operators.
\end{abstract}

Keywords: Nonlinear Resonance, Diatomic Molecule, Quantum Resonances

\section{Introduction}

The study of quantum dynamics in the interval of parameters where classical chaotic behavior occurs is what we call "Quantum Chaos, Chaology, or Quantum Manifestation of Chaos" [1] which deals with some type of quantum manifestation of the classical chaos, mainly associated with the statistical properties of near neighbor levels of energy of the system [1]. In contrast, for quantum systems associated to non chaotic classical ones, it is mostly believed that classical dynamical behavior must occur for large quantum numbers or high value of the action variable [2] (Rydberg states). In particular, studies of dynamical chaos in atomic and molecular systems has been of great theoretical and practical interest [3-12] since not enough integrals of motion are found either in the classical or in its quantum system. Different approaches and studies have been used for the classical [13-15] and quantum (quasi-classical region) [16-18] cases, and most of them are based on the Morse potential as the inter-atomic interaction [19]. On the other hand, the classical study of the dynamics of atomic and molecular systems has shown that, under certain conditions, these systems are capable of exhibiting a chaotic behavior, even in the case the system has few degrees of free- dom. In what follows of this introduction and to have a better perspective of the problem, we summarize what Berman et al. [20] did for the classical part of the problem.

It is known that the dynamics of a diatomic molecule in a resonant circularly polarized electric field can be modeled by the Hamiltonian

$$
\begin{aligned}
H\left(I, \vartheta ; p_{\theta}, \theta ; p_{\phi}, \varphi\right)= & H_{0}^{(\text {vib })}(I, \vartheta)+H_{0}^{(\text {rot })}\left(p_{\theta}, \theta ; p_{\phi}, \varphi\right) \\
& +H_{\text {int }}(I, \vartheta, \theta, t)
\end{aligned}
$$

where $H_{0}^{(v i b)}$ describes the vibration of the molecule along its axis in terms of the action variable $I$ and its conjugated angle variable $\vartheta, H_{0}^{(r o t)}$ describes the rotation of the molecule around its transversal axis of symmetry in terms of spherical coordinates $(r, \theta, \varphi)$, and $H_{\text {int }}=-\boldsymbol{d} \cdot \boldsymbol{E}(t)$ describes the interaction of the electric dipole moment $(\boldsymbol{d})$ of the molecule with and external electric field $(\boldsymbol{E}(t))$. These term are given explicitly by

$$
\begin{aligned}
& H_{0}^{(\text {vib })}(I, \vartheta)=\omega_{e} I-\hbar x_{e} \omega_{e} I^{2}, \\
& H_{0}^{(\text {rot })}\left(p_{\theta}, \theta ; p_{\phi}, \varphi\right)=\frac{1}{2 \mu r^{2}}\left(p_{\theta}^{2}+\frac{p_{\phi}^{2}}{\sin ^{2} \theta}\right),
\end{aligned}
$$




$$
\begin{aligned}
H_{i n t}= & -E_{0} d_{0}-\frac{E_{0} e_{e f f}}{2} \sqrt{\frac{2 I}{\mu \omega_{e}}} \sin \theta[\cos (\vartheta-\varphi+\omega t) \\
& +\cos (\vartheta+\varphi-\omega t)] .
\end{aligned}
$$

For the derivation of these expressions, the motion of the molecule has been done with respect the center of mass $R=\left(m_{1} \boldsymbol{r}_{1}+m_{2} \boldsymbol{r}_{2}\right) /\left(m_{1}+m_{2}\right)$ and relative $\boldsymbol{r}=\boldsymbol{r}_{2}-\boldsymbol{r}_{1}$ coordinates associated to the diatomic molecule, where $\mu$ is its reduced mass. The parameter $x_{e}$ is defined as $x_{e}=\left(\hbar / 2 \omega_{e}\right)\left(d^{2} H_{0}^{(v i b)} / d I^{2}\right)$. The electric field has been chosen as $\boldsymbol{E}(t)=E_{0}(\cos \omega t, \sin \omega t, 0)$, and the magnitude of the electric dipole moment has been given by $d=d_{0}+e_{\text {eff }} \sqrt{2 I / \mu \omega_{e}} \cos \vartheta$, where $d_{0}=e_{\text {eff }} r_{0}$, being $e_{\text {eff }}$ the effective charge of the molecule and $r_{0}$ represents the point of the minimum on the Morse potential [19] which simulates the atom-atom interaction in the diatomic molecule and has been taken up to second order.
The average small vibration oscillation around the equilibrium point $r_{o}$ is just $\int_{0}^{2 \pi}(\Delta r)^{2} d \vartheta=2 I \cos ^{2} \vartheta / \mu \omega_{e}$, with $\omega_{e}$ representing the angular frequency of the oscillation of the molecule at first order. The dynamical system described by this Hamiltonian close to resonance $\left(\omega \approx \omega_{e}\right)$, and under the condition $\omega>>|\dot{\varphi}|,|\dot{\theta}|$ has the following constant of motion

$$
p_{\varphi}-I=\text { constant }=\hbar k,
$$

The Hamiltonian (1.1) can be written in a more suitable form through a change of variable defined by the generatrix function $F_{2}(\vartheta, \phi, \theta ; n, k, p ; \tau)=\hbar(n+1 / 2)$ $(\vartheta+\varphi-\omega \tau / \Omega)+\hbar k \varphi+\hbar p \theta$, which are given by

$$
\begin{gathered}
\psi=\vartheta+\varphi-\omega t, \quad \tilde{\theta}=\theta, \quad \tilde{\varphi}=\varphi \\
I=\hbar(n+1 / 2), \quad p_{\theta}=\hbar p, \quad p_{\varphi}=\hbar k+I, \quad \tau=\Omega t,
\end{gathered}
$$

and the Hamiltonian in this coordinates is written as

$$
\tilde{H}(N, \psi ; P, \theta ; K, \varphi)=\hbar\left(\omega_{e}-\omega\right)\left(n+\frac{1}{2}\right)-\hbar x_{e} \omega_{e}\left(n+\frac{1}{2}\right)^{2}+\beta\left[p^{2}+\frac{1}{\sin ^{2} \theta}\left(k+n+\frac{1}{2}\right)^{2}\right]-\frac{W}{2} \sqrt{n+\frac{1}{2}} \sin \theta \cos \psi
$$

where the variable " $n$ " is assumed to have continuous values, and the following definitions have been made: $\beta=\hbar^{2} / 2 \mu r_{0}^{2}$, and $W=E_{0} e_{\text {eff }} \sqrt{2 \hbar / \mu \omega_{e}}$. This Hamiltonian depends only in the conjugate variables $(n, \psi)$ and $(p, \theta)$ since $\varphi$ is an ignorable variable, and therefore, $k$ is a constant of motion. In this way, the Hamilton equations with this variables define the four dimensional classical dynamical system

$$
\begin{aligned}
& \frac{\mathrm{d} n}{\mathrm{~d} \tau}=-\frac{W}{2} \sqrt{n+\frac{1}{2}} \sin \theta \sin \psi, \\
& \frac{\mathrm{d} \psi}{\mathrm{d} \tau}=\hbar\left(\omega_{e}-\omega\right)-2 \hbar x_{e} \omega_{e}\left(n+\frac{1}{2}\right)+\frac{2 \beta}{\sin ^{2} \theta}\left(k+n+\frac{1}{2}\right)-\frac{W}{4 \sqrt{n+1 / 2}} \sin \theta \cos \psi \\
& \frac{\mathrm{d} p}{\mathrm{~d} \tau}=\frac{2 \beta}{\sin ^{3} \theta}\left(k+n+\frac{1}{2}\right)^{2} \cos \theta+\frac{W}{2} \sqrt{n+\frac{1}{2}} \cos \theta \cos \psi, \\
& \frac{\mathrm{d} \theta}{\mathrm{d} \tau}=2 \beta p .
\end{aligned}
$$

This system has its critical points at $p=0, \theta=\pi / 2$, $\psi=m \pi$ with $m \in Z$, and $n=n_{i}$ with $i=1,2,3$ the roots of a third order polynomial. In the example given by the reference [20], the parameters associated to the diatomic molecule $\mathrm{GeO}$ [17] are used,

$$
\begin{gathered}
\hbar \omega_{e}=985.8 \mathrm{~cm}^{-1}, \hbar\left(\omega_{e}-\omega\right)=15 \mathrm{~cm}^{-1}, \\
\hbar x_{e} \omega_{e}=2.2 \mathrm{~cm}^{-1}, \beta=0.48 \mathrm{~cm}^{-1}, \\
d_{0}=3.28 \mathrm{D}, r_{0}=1.62 \AA, \mu=13.1 \mathrm{amu}
\end{gathered}
$$

where the units have been selected such that $\hbar \Omega=\frac{3}{2} k_{b} T=1 \mathrm{~cm}^{-1}$ and $T=0.956 \mathrm{~K}$. Note that these parameters correspond to be close to resonance. Then, there is one center points at $\psi=\pi$ and $n_{1} \approx 2$ which forms the first resonances of the system (for $W=0.05$ $\mathrm{cm}^{-1}$ and $k=0$ ), and there is other resonance located near $n_{2} \approx 1.5$ which is due to $\theta$ degree of freedom rather that a critical point of the system. The resonances overlapping Chirikov's criterion [13] for appearing of chaos was verified at $W=0.177 \mathrm{~cm}^{-1}$, and total chaotic behavior is observed after $W=1.03 \mathrm{~cm}^{-1}$, see Figure 1 . This result suggests that classical chaotic behavior appears within the first two exited states of the associated quantum system. However, the correspondence principle [2] tells us that the quasi-classical behavior of a quantum system is gotten for very large quantum numbers. Therefore, one would not expect any quasi-classical behavior for ground and first exited states of the quantum system. 


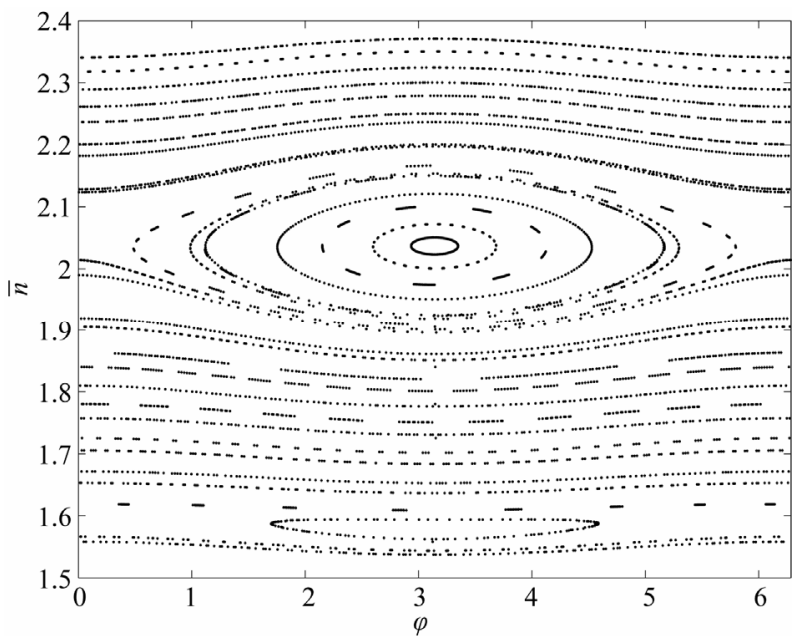

(a)

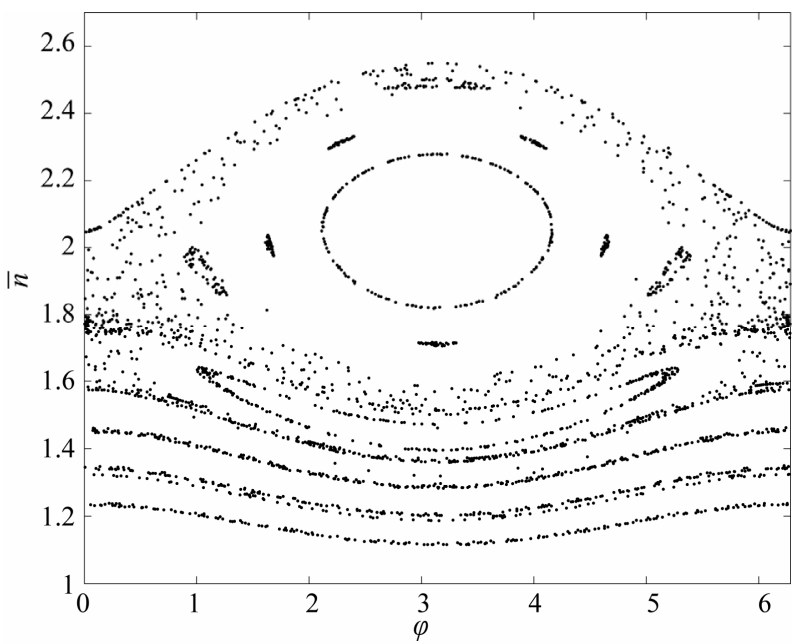

(b)

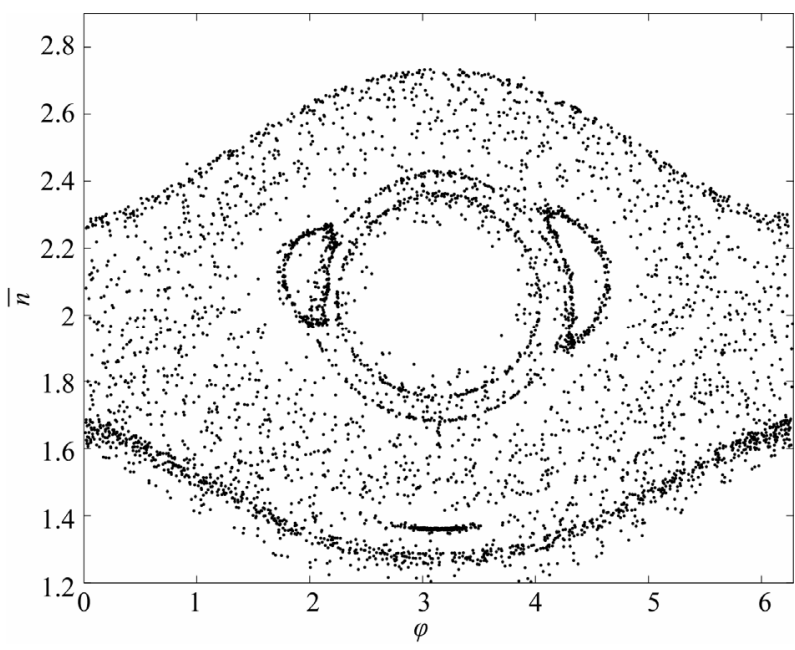

(c)

Figure 1. Poincaré map for $\theta=\pi / 2$ with $\theta>0, k=0, \theta_{0}=1$, and $p_{0}=0$ and for: (a) $W=0.048 \mathrm{~cm}^{-1}$; (b) $W=0.68 \mathrm{~cm}^{-1}$; (c) $W=1.03 \mathrm{~cm}^{-1}$.
In this way, in this paper we study the quantum behavior of this system in the region of parameters where this classical chaotic dynamics appears. This behavior could be important in the study of diatomic molecular clouds during the star born formation or supernova wind shock studies from dying stars $[25,26]$. We solve the associated Schrödinger equation, assuming the wave function is a linear combination of the stationary states with time depending coefficients and solving numerically the resulting equations for these coefficients, and picture the expectation values of the quantum variables in a phase space-like to look for a similarity with the classical behavior.

\section{Quantum Dynamics}

\subsection{Quantum Hamiltonian}

Our goal is to solve the Schrödinger equation,

$$
i \hbar \frac{\partial}{\partial t}|\Psi(t)\rangle=\hat{H}|\Psi(t)\rangle,
$$

where $\hat{H}$ is the Hermitian operator associated to the Hamiltonian,

$$
\begin{aligned}
H= & \omega_{e} I-\hbar x_{e} \omega_{e} I^{2}+\frac{1}{2 \mu r_{0}^{2}}\left(p_{\theta}^{2}+\frac{p_{\varphi}^{2}}{\sin \theta^{2}}\right) \\
& -\frac{E_{0} e_{e f f}}{2} \sqrt{\frac{2 I}{\mu \omega_{e}}} \sin \theta \cos (\vartheta+\varphi-\omega t) .
\end{aligned}
$$

For this propose, the following operators are assigned to the observables,

$$
\begin{gathered}
I \rightarrow \hbar\left(a a^{\dagger}+\frac{1}{2}\right), \quad \vartheta \rightarrow \hat{\vartheta}, \\
L^{2}=\left(p_{\theta}^{2}+\frac{p_{\varphi}^{2}}{\sin ^{2} \theta}\right) \rightarrow \hat{L}^{2}, \quad \theta \rightarrow \hat{\theta}, \quad \varphi \rightarrow \hat{\varphi}
\end{gathered}
$$

where $a$ and $a^{\dagger}=(a)^{\dagger}$ represent the usual ascend and descend operators of the quantum harmonic oscillator. If $\{|n\rangle\}$ and $\{|l m\rangle\}$ are the basis for the harmonic oscillator and the angular moment operators such that

$$
\begin{aligned}
& a|n\rangle=\sqrt{n}|n\rangle, \quad a^{\dagger}|n\rangle=\sqrt{n}|n+1\rangle, \\
& a^{\dagger} a|n\rangle=\hat{n}|n\rangle=n|n\rangle, \quad\left[a, a^{\dagger}\right]=1,
\end{aligned}
$$

and

$$
\begin{gathered}
\hat{L}^{2}|\operatorname{lm}\rangle=\hbar^{2} l(l+1)|\operatorname{lm}\rangle, \\
\hat{L}_{z}|\operatorname{lm}\rangle=\hbar m|l m\rangle, \quad-l \leq m \leq l
\end{gathered}
$$

the action of $\hat{\vartheta}$ is defined in terms of the phase operator [21-23] as 


$$
\begin{gathered}
\mathrm{e}^{-i \hat{\vartheta}}:=\frac{1}{\sqrt{a a^{\dagger}}} a, \quad \mathrm{e}^{i \hat{\vartheta}}:=\left(\mathrm{e}^{-i \hat{\vartheta}}\right)^{\dagger}=a^{\dagger} \frac{1}{\sqrt{a a^{\dagger}}}, \\
\cos \hat{\vartheta}:=\frac{1}{2}\left(\mathrm{e}^{i \hat{\vartheta}}+\mathrm{e}^{-i \hat{\vartheta}}\right), \quad \sin \hat{\vartheta}:=\frac{1}{2}\left(\mathrm{e}^{i \hat{\vartheta}}-\mathrm{e}^{-i \hat{\vartheta}}\right) .
\end{gathered}
$$

These operators have the following properties,

$$
\begin{aligned}
{[\hat{n}, \cos \hat{\vartheta}] } & =i \sin \hat{\vartheta}, \quad[\hat{n}, \sin \hat{\vartheta}]=-i \cos \hat{\vartheta}, \\
\mathrm{e}^{i \hat{\vartheta}}|n\rangle & =|n+1\rangle, \quad \mathrm{e}^{-i \hat{\vartheta}}|n\rangle=|n-1\rangle .
\end{aligned}
$$$$
\hat{W}=-\frac{W}{2} \sin \hat{\theta}\left\{\sqrt{\hat{n}+\frac{1}{2}}[\cos \hat{\vartheta} \cos (\hat{\varphi}-\omega t)-\sin \hat{\vartheta} \sin (\hat{\varphi}-\omega t)]+[\cos \hat{\vartheta} \cos (\hat{\varphi}-\omega t)-\sin \hat{\vartheta} \sin (\hat{\varphi}-\omega t)] \sqrt{\hat{n}+\frac{1}{2}}\right\} .
$$

To construct the Hermitian operator $\hat{W}$, we have used the fact that for any operator $\hat{A}$, the operator $\left(\hat{A}+\hat{A}^{\dagger}\right) / 2$ is Hermitian. Using the commutation relations of Equation (16), one gets the commutativity of the vibrational and rotational operators, $\left[f\left(a, a^{\dagger}\right), g(\hat{\theta}, \hat{\varphi})\right]=0$, and we see that $\hbar \hat{k}=\hat{L}_{z}-\hbar(\hat{n}+1 / 2)$ is a constant of motion, that is

$$
[\hat{H}, \hbar \hat{k}]=\left[\hat{H}, \hat{L}_{z}-\hbar\left(\hat{n}+\frac{1}{2}\right)\right]=0
$$

which is the quantum analogue of Equation (4) and has the physical meaning that external electric field's photons excite the rotational and vibrational degree of freedom with the same number of quanta. The main reason for choosing the phase operator as Equation (15) was to be able to get this quantum constant of motion correctly.

\subsection{Time Evolution Equations}

Using the number states $|n\rangle$ and the spherical harmonic states $|\operatorname{lm}\rangle$, we see that $\hat{H}_{0}$ is diagonal in the basis $\{|n l m\rangle=|n\rangle \otimes|\operatorname{lm}\rangle\}$, and the eigenvalues are given by
From Equation (11) and the definitions (12) - (14), we get the quantum Hamiltonian of the form $\hat{H}=\hat{H}_{0}+\hat{W}$, where $\hat{H}_{0}$ and $\hat{W}$ are given by

$$
\begin{aligned}
\hat{H}_{0}= & \hbar \omega_{e}\left(a^{\dagger} a+\frac{1}{2}\right)-\hbar x_{e} \omega_{e}\left(a^{\dagger} a+\frac{1}{2}\right)^{2} \\
& +\frac{\beta}{\hbar^{2}} \hat{L}^{2}
\end{aligned}
$$

and

$$
\begin{aligned}
& \hat{H}_{0}|n l m\rangle=E_{n l}|n l m\rangle, \\
& E_{n l}=\hbar \omega_{e}\left(n+\frac{1}{2}\right)-\hbar x_{e} \omega_{e}\left(n+\frac{1}{2}\right)^{2}+\beta l(l+1),
\end{aligned}
$$

where there is a $(2 l+1)$ degeneration due to quantum number " $m$ ". On the other hand, since the relation $E_{n+1, l}-E_{n l}>0$ must be satisfied, the quantum vibrational number " $n$ " is bounded [19] in the following way

$$
0 \leq n \leq\left[\frac{1}{2 x_{e}}-\frac{1}{2}\right]
$$

where $[x]$ means the integer part of the real number " $x$ ". Therefore, the spectrum is finite. Let us propose the solution of Equation (10) of the form

$$
|\Psi(t)\rangle=\sum_{n l m}^{\max } D_{n l m}(t) e^{-i t E_{n l} / \hbar}|n l m\rangle .
$$

Now, substituting this equation in (10) and using the orthogonality relation $\left\langle n^{\prime} l^{\prime} m^{\prime} \mid n l m\right\rangle=\delta_{n^{\prime} n} \delta_{l^{\prime} l} \delta_{m^{\prime} m}$, we get the system of equations for the coefficients as

$$
i \hbar \dot{D}_{n^{\prime} l^{\prime} m^{\prime}}=\sum_{n l m}^{\max } e^{i t\left(E_{n^{\prime} l^{\prime}}-E_{n l}\right) / \hbar} D_{n l m} \hat{W}_{n^{\prime} l^{\prime} m^{\prime} ; n l m},
$$

where the matrix elements $W_{n^{\prime} l^{\prime} m^{\prime}, n l m}=\left\langle n^{\prime} l^{\prime} m^{\prime}|\hat{W}| n l m\right\rangle$ are given by

$$
\begin{aligned}
W_{n^{\prime} I^{\prime} m^{\prime}, n l m}= & -\frac{W}{2}\left\{\left[\left\langle n^{\prime}\left|\sqrt{\hat{n}+\frac{1}{2}} \cos \hat{\vartheta}\right| n\right\rangle+\left\langle n^{\prime}\left|\cos \hat{\vartheta} \sqrt{n}+\frac{1}{2}\right| n\right\rangle\right]\left\langle l^{\prime} m^{\prime}|\sin \hat{\theta} \cos (\hat{\varphi}-\omega t)| \operatorname{lm}\right\rangle\right. \\
& \left.-\left[\left\langle n^{\prime}\left|\sqrt{\hat{n}+\frac{1}{2}} \sin \hat{\vartheta}\right| n\right\rangle+\left\langle n^{\prime}\left|\sin \hat{\vartheta} \sqrt{\hat{n}+\frac{1}{2}}\right| n\right\rangle\right]\left\langle l^{\prime} m^{\prime}|\sin \hat{\theta} \sin (\hat{\varphi}-\omega t)| \operatorname{lm}\right\rangle\right\} .
\end{aligned}
$$

From the expressions A1 to A7 of the Appendix, this matrix elements can be written as

$$
W_{n^{\prime} l^{\prime} m^{\prime}, n l m}=-\frac{W}{4}\left(\sqrt{n+1 / 2}+\sqrt{n^{\prime}+1 / 2}\right)\left(\delta_{l^{\prime}}^{l+1}-\delta_{l^{\prime}}^{l-1}\right) \times\left(e^{-i \omega t} \delta_{n^{\prime}}^{n+1} \delta_{m^{\prime}}^{m+1} \frac{C_{l^{\prime} m^{\prime}}}{C_{l m}(2 l+1)}-e^{i \omega t} \delta_{n^{\prime}}^{n-1} \delta_{m^{\prime}}^{m-1} \frac{c_{l m}}{C_{l^{\prime} m^{\prime}}\left(2 l^{\prime}+1\right)}\right) \text {. }
$$

Substituting this expression in Equation (23) and using the same dimensionless variables defined in the intro- duction, we get the time evolution equation of the coefficients as 


$$
\begin{aligned}
& i D_{n^{\prime} l^{\prime} m^{\prime}}^{\prime}=-\frac{W}{4}\left[(\sqrt{n+1 / 2}+\sqrt{n-1 / 2}) \frac{c_{l m} \mathrm{e}^{i \tau \Omega_{n l,(-),(-)}}}{c_{l-1, m-1}(2 l-1)} D_{n-1, l-1, m-1}-(\sqrt{n+1 / 2}+\sqrt{n-1 / 2}) \frac{c_{l m} \mathrm{e}^{i \tau \Omega_{n l,(-),(+)}} D_{l+1, m-1}(2 l+3)}{c_{n-1, l+1, m-1}}\right. \\
& \left.-(\sqrt{n+1 / 2}+\sqrt{n+3 / 2}) \frac{C_{l-1, m+1} \mathrm{e}^{i \tau \Omega_{n l,(+),(+)}}}{C_{l m}(2 l+1)} D_{n+1, l-1, m+1}+(\sqrt{n+1 / 2}+\sqrt{n+3 / 2}) \frac{C_{l+1, m+1} \mathrm{e}^{i \tau \Omega_{n l,(+),(-)}}}{C_{l m}(2 l+1)} D_{n+1, l+1, m+1}\right] \text {, }
\end{aligned}
$$

where we have made the definitions $D^{\prime}=\mathrm{d} D / \mathrm{d} \tau$, $\Omega_{n l,( \pm),(-)}=E_{n \pm 1, l-1}-E_{n l} \mp \hbar \omega$, and

$\Omega_{n l,( \pm),(+)}=E_{n \pm 1, l+1}-E_{n l} \mp \hbar \omega$. The selection rules deduced from (26) are

$$
\Delta l= \pm 1, \Delta n= \pm 1 \text { and } \Delta m= \pm 1 .
$$

Note that the last two terms of these expressions are a consequence of the constant of motion (19). The time evolution of the coefficients in Equation (27) and the selection rules in Equation (25) are similar to the electric dipole transitions in an atom, except with the extra selection rule of $n$. Furthermore, suppose we are initially in a given state $|\Psi(0)\rangle=\left|n_{0} l_{0} m_{0}\right\rangle$ and we set the frequency $\omega$ to be such that it is almost in resonance with the frequency of an allowed transition, say $\left|n_{f} l_{f} m_{f}\right\rangle$ (that is $\left.\hbar \omega-E_{n_{f}, l_{f}}-E_{n_{0}, l_{0}}\right)$. For this case and neglecting the non-resonant transitions, the equations of motion (28) becomes

$$
i D_{0}^{\prime}=\alpha e^{i \tau \Omega_{r}} D_{f} \text { and } i D_{f}^{\prime}=\alpha e^{-i \tau \Omega_{r}} D_{0},
$$

where $\alpha$ and $\Omega_{r}$ are defined as

$$
\alpha=-\left(\sqrt{n_{0}+1 / 2}+\sqrt{n_{f}+1 / 2}\right) W c_{l_{0}, m_{0}} / 4 c_{l_{f}, m_{f}}\left(2 l_{f}+1\right)
$$

and $\Omega_{r}=E_{n_{f}, l_{f}}-E_{n_{o}, l_{o}}-\hbar \omega$. In matrix notation, Equation (26) is written as

$$
i \frac{\mathrm{d}}{\mathrm{d} \tau}\left(\begin{array}{c}
D_{0} \\
D_{f}
\end{array}\right)=\alpha\left(\begin{array}{cc}
0 & e^{-i \Omega \tau} \\
e^{i \Omega \tau} & 0
\end{array}\right)\left(\begin{array}{c}
D_{0} \\
D_{f}
\end{array}\right)
$$

which in terms of the Pauli operators becomes

$$
\begin{aligned}
& i \frac{\mathrm{d}}{\mathrm{d} \tau}|\psi\rangle=\alpha\left[\cos \Omega_{r} \tau \hat{\sigma}_{x}+\sin \Omega_{r} \tau \hat{\sigma}_{y}\right]|\psi\rangle, \\
& \text { with }|\psi\rangle=\left(\begin{array}{c}
D_{0} \\
D_{f}
\end{array}\right),
\end{aligned}
$$

and this one is of the form

$$
\begin{aligned}
i \frac{\mathrm{d}}{\mathrm{d} \tau}|\psi\rangle & =\hat{H}_{a t}|\psi\rangle, \\
\text { with } \hat{H}_{a t} & =\alpha\left[\cos \Omega_{r} \tau \hat{\sigma}_{x}+\sin \Omega_{r} \tau \hat{\sigma}_{y}\right],
\end{aligned}
$$

which is the Schrödinger equation for a two level atom introduced in a circularly polarized electromagnetic field [5].

\subsection{Numerical Results}

We solve numerically Equation (28), considering only the coefficients $D_{n l m}$ for $n, l \leq 3$. We use the same parameters used in the classical numerical calculations, Equation (9), which implies to have a close resonant transition between the states $|100\rangle$ and $|211\rangle$ with $\left|\Omega_{10,(+),(+)}\right|=0.82$. Higher order of excitation are not considered since we want to see what happen to the states closely related with the classical ones, where classical chaos appears. In this way, we are not interested here in the quasi-classical region (very highly exited states) but in the deep quantum region (first few states), where quantum-classical correspondence is not expected. The results of the numerical simulations are shown in Figures 2(a) and (b) which represent the typical oscillation between the population in the two resonant states. The Figure 2(a) shows the transition probabilities for small values of $W$ before there is a considerable mixing of states (observe that $\left|c_{211}\right|^{2}<0.5$ ). Figure 2(b) shows the same probabilities but for the value

$W=1.03 \mathrm{~cm}^{-1}$ which should correspond to have classical chaotic behavior, in the classical dynamics (note that the value of $W$ for classical chaos to appear is $\left.W_{c h}=0.177 \mathrm{~cm}^{-1}\right)$.

We see also that the classical value of the closed classical resonance suggests overlapping between quantum states in $n=1$ and $n=2$, as we precisely observe in our simulations, which is consequence of the resonant transition frequency between the states $|100\rangle$ and $|211\rangle$.

\subsection{Quantum Phase Space Pictures}

In this section we try two different approaches to see a better relation between the quantum and classical dynamics. Here, we are interested in the behavior of expectation values of the dynamical variables rather than the statistical properties in the phase space due to the Schrödinger wave function (Wigner [27], Husimi [28], or Glauber-Sudarshan [29,30] distribution functions) since these values are the ones we want to compare. The first and most used approach [21-23], is to use the phase space representation in terms of the expectation value of the dimensionless canonical variables $\hat{X}$ and $\hat{P}$

$$
\hat{X}=\frac{a+a^{\dagger}}{2}, \quad \hat{P}=\frac{a-a^{\dagger}}{2 i} \text {. }
$$



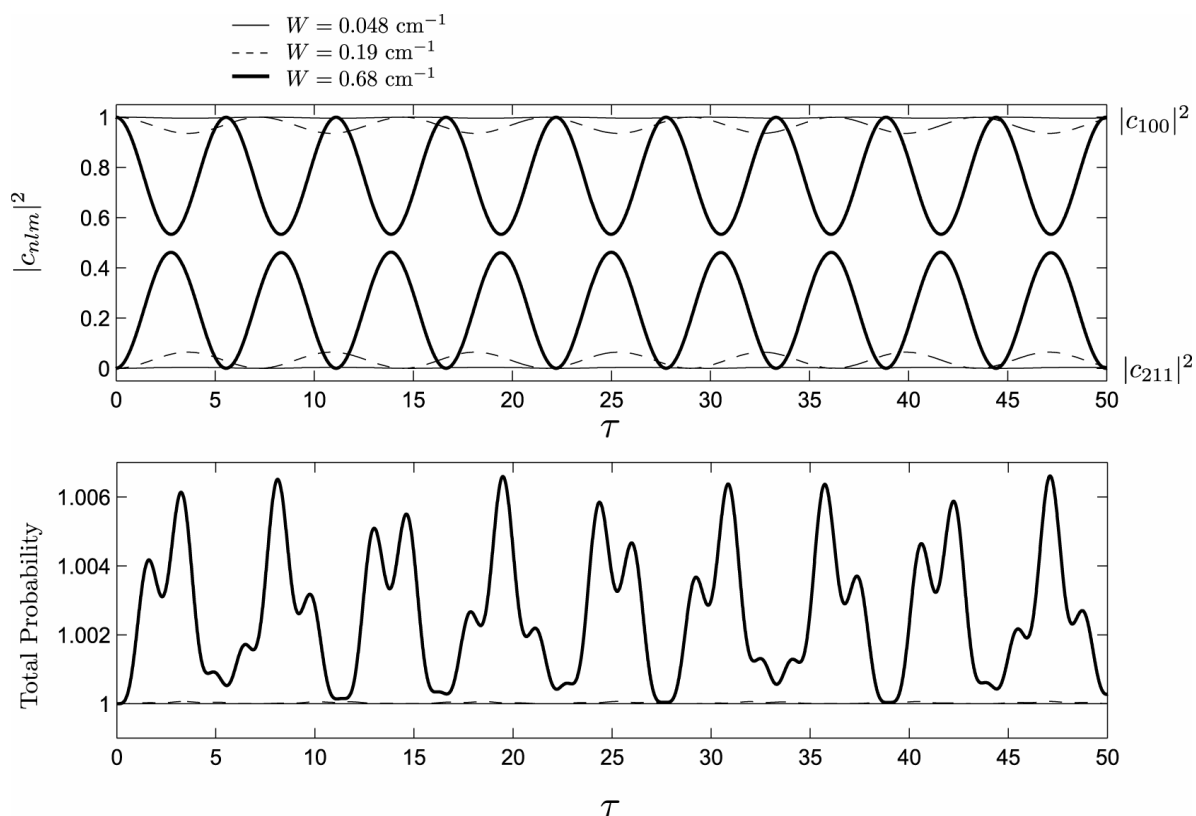

(a)
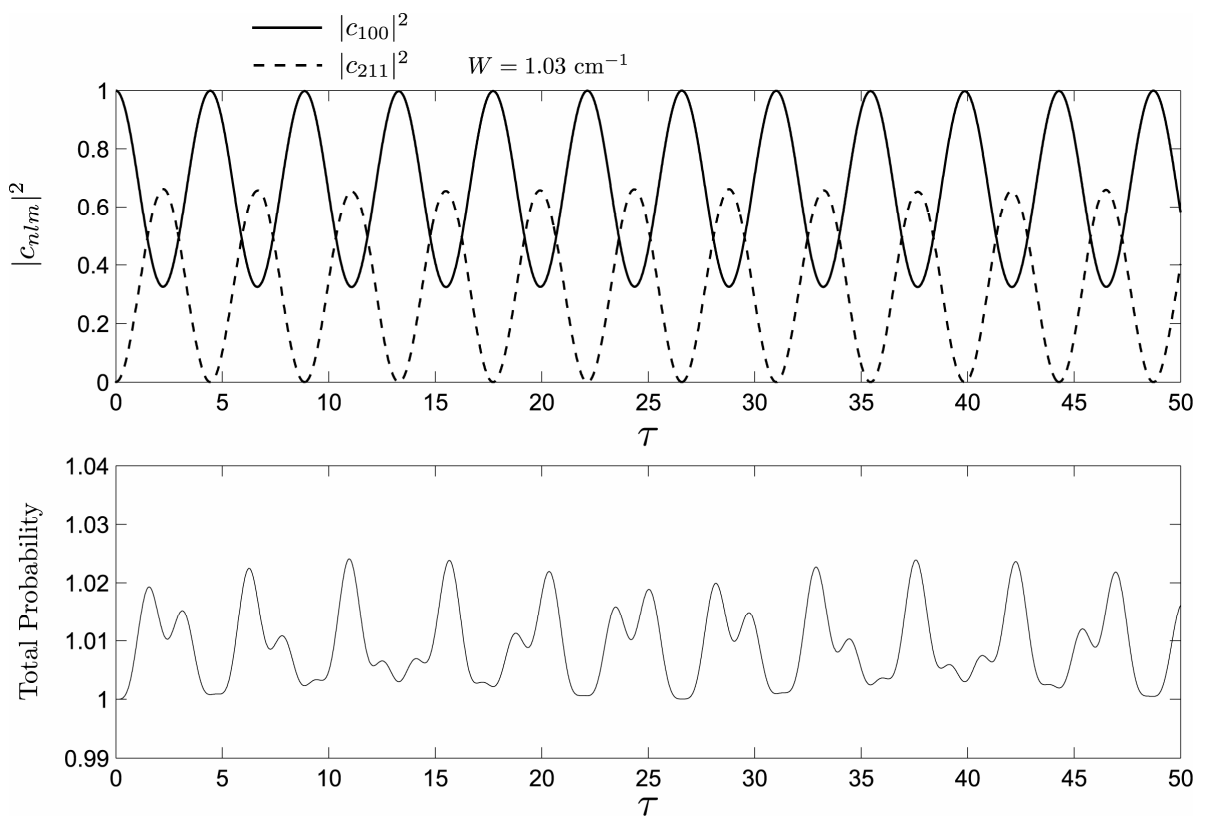

(a)

Figure 2. Time evolution of the total probability, the probability amplitude of the state $|100\rangle$ (upper line) and the state $|211\rangle$ (lower line) for different values of the perturbation: (a) $W=0.048 \mathrm{~cm}^{-1}$, solid; $W=0.19 \mathrm{~cm}^{-1}$, dashed; $W=0.68 \mathrm{~cm}^{-1}$, bold; (b) $W=1.03 \mathrm{~cm}^{-1}$.

The results of the numerical simulations of this approaches is presented in Figure 3, where we used the same parameters of expression (15) and $W=1.03 \mathrm{~cm}^{-1}$. For the initial state wave function of the system we chose a poisson-like distribution in the coefficients $D_{n l 0}$ with the maximum in value in the state $D_{100}$. This initial state is determined by the following coefficients

$$
\begin{aligned}
& D_{000}=\sqrt{\frac{1.5}{12}}, D_{010}=\sqrt{\frac{0.2}{12}}, D_{020}=\sqrt{\frac{0.05}{12}} \\
& D_{100}=\sqrt{\frac{8}{12}}, D_{110}=\sqrt{\frac{0.4}{12}}, D_{120}=\sqrt{\frac{0.1}{12}} \\
& D_{200}=\sqrt{\frac{1.5}{12}}, D_{210}=\sqrt{\frac{0.2}{12}}, D_{220}=\sqrt{\frac{0.05}{12}} .
\end{aligned}
$$



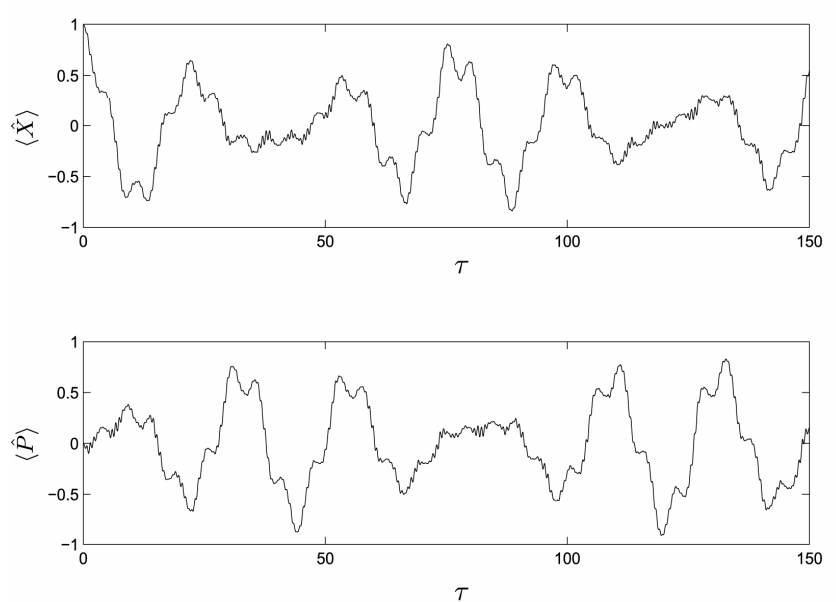

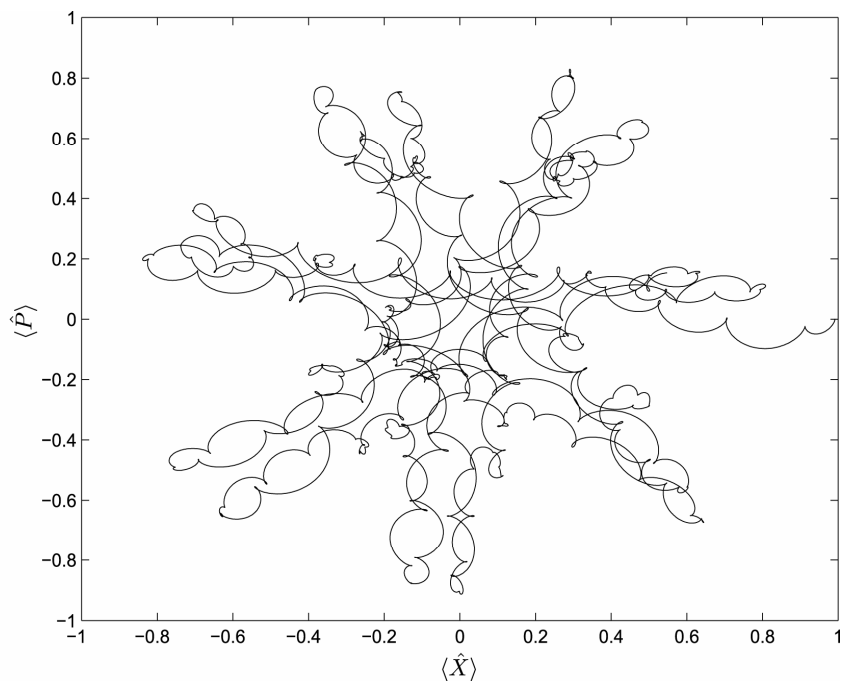

$\langle\hat{X}\rangle$

Figure 3. Phase space like picture with the expectation values variable $\langle X\rangle$ and $\langle P\rangle$ for the initially poission-like distribution, Equation (29), using the same parameters as with the classical case.

The reason to use this initial state is based in the properties of the coherent states of the harmonic oscillator. This selection not only gives a well defined initial value of the expectation values, but also permits a further study in terms of the Liouville dynamics for both the classical and quantum case [24]. Although the dynamics of each variable seem to be stable and similar to each other, the phase space representation does not seem to give any picture alike to the classical dynamics of the system (see reference [20]), i.e., the phase space in terms of the canonical variables $\langle\hat{X}\rangle$ and $\langle\hat{P}\rangle$ shows no resemblance to the classical dynamics, and this happen for other different initial states.

In the second approach, we will use the phase space representation in terms of the expectation value of $\arg \left(\mathrm{e}^{i \hat{\vartheta}}\right)$, as the angle, and the expectation value of the number operator $\hat{n}$. In polar coordinates, the $\langle\hat{n}\rangle$ will correspond to the radius and $\left\langle\arg \left(\mathrm{e}^{i \hat{\vartheta}}\right)\right\rangle$ to the angle of the phase space of this set of variables, $\hat{n}=a^{\dagger} a$ and $\mathrm{e}^{i \hat{\vartheta}}=a^{\dagger}\left(1 / \sqrt{a a^{\dagger}}\right)$. The expectation values of these variables represent the classical analogous of the variables on Figure 1 above. In the upper left plot of Figure 4 it is shown the time evolution of $\langle n\rangle$ which resemblances to the classical case in terms of the main and different frequencies with which it oscillates. For the numerical simulations results presented in these figures we used the same parameters as in the Subsection 2.3, and the same initial state (29). The phase space picture in terms of the operators $\hat{n}$ and $e^{i \hat{\vartheta}}$ seems to have a little bit resemblance with the classical results, perhaps because the dynamics of $\hat{n}$ resembles the classical part. Also, the sudden slow changes of $\arg \left(e^{i \hat{\vartheta}}\right)$ seem to suggest some kind of relation with the resonances of the classical case.

\section{Conclusions and Comments}

We have studied the quantization of a diatomic molecule by solving the Schrödinger equation with the known Hamiltonian of the diatomic molecule with a circularly polarized resonant rf-field, written in spherical coordinates (rotations) and angle-action variables (vibrations). The wave function was expanded in a finite combination of a proper stationary basis with time dependent coefficients, and the system of equations for these coefficients was obtained. Using the same parameters as in the classical case, a near resonant transition between the states $|100\rangle$ and $|211\rangle$ is gotten, which correspond to the closer integer numbers for $n$ where the classical non linear resonances appeared, $n_{1} \approx 2$ and $n_{2} \approx 1.5$. Using a poisson-like distributed initial wave function in the quantum numbers, with maximum in the resonant state $|100\rangle$, we try two different approaches to see the quantum phase space expectation value dynamics and compare it with the classical case. The usual approach, using the canonical variables $\hat{X}$ and $\hat{P}$, fails to provide any intuitive picture of the classical case. On the other hand, the approach using the expectation value of $e^{i \hat{\varphi}}$ and $\hat{n}$ suggests some resemblance and relationship with the classical case. Therefore, we have here the following situation, on one hand, the correspondence principle tells us that we must have the quasi-classical behavior (classical chaos) for this quantum system at very large quantum number. However, classical chaotic behavior is obtained just for the associated first states of the quantum system, implying that quasi-classical chaotic behavior can not be obtained here. So, as one could expect for this case, quantum dynamics does not follow the classical one. 

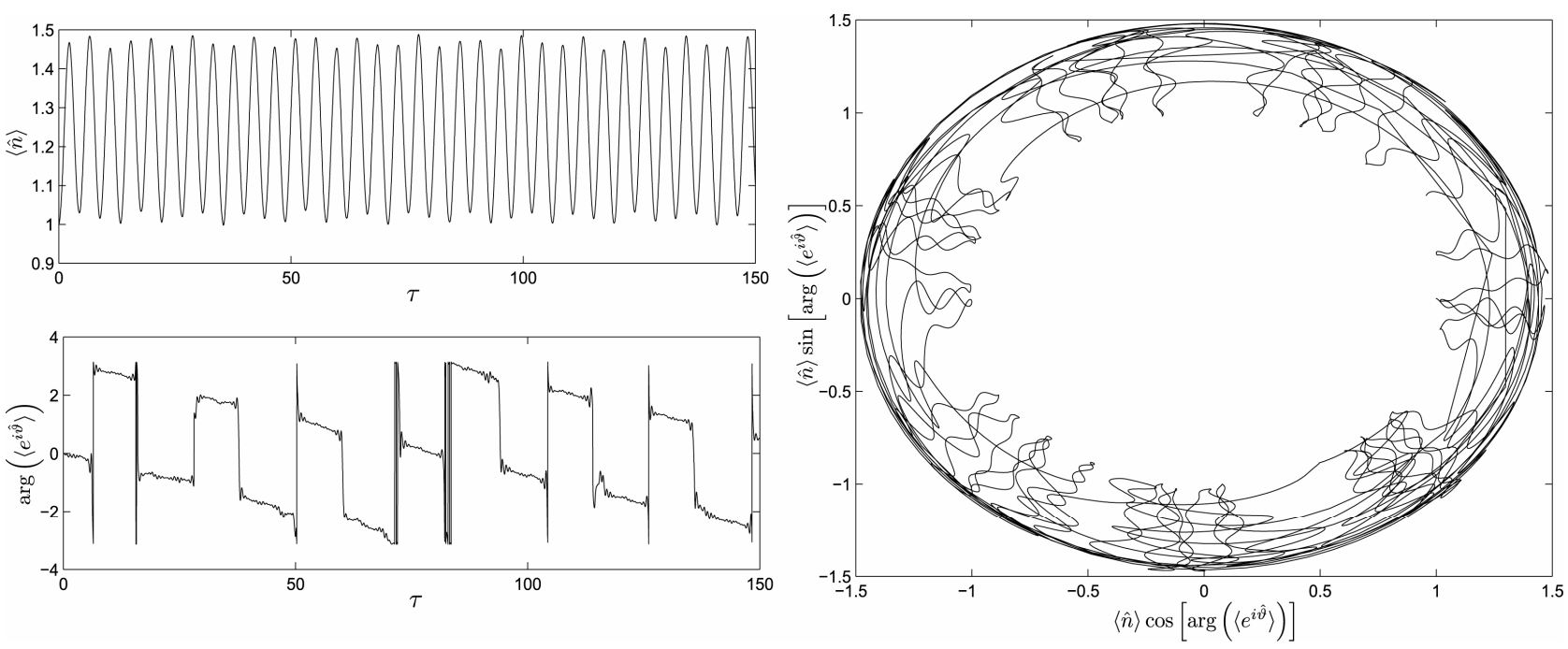

Figure 4. Phase space like picture with the same parameters as in the classical case in terms of the expectation values $\langle\hat{n}\rangle$ and $\left\langle\arg \left(e^{i \hat{\vartheta}}\right)\right\rangle$.

\section{Acknowledgements}

We want to thank Professor Gennady P. Berman for his comments and suggestions about this subject, and CONACYT for its support with the grand number 0104129.

\section{References}

[1] E. R. Linda, "The Transition to Chaos," Springer-Verlag, New York, 2004.

[2] A. Messiah, "Quantum Mechanics I," John Wiley \& Sons, New York, 1964.

[3] A. J. Lichtenberg and M. A. Liberman, "Regular and Stochastic Motion," Springer-Verlag, Berlin, 1983.

[4] G. Casati, B. V. Chirikov, D. L. Shepelyansky and I. Guarnery, "Relevance of Classical Chaos in Quantum Mechanics: The Hydrogen Atom in Monochromatic Field," Physics Reports, Vol. 154, No. 2, 1983, pp. 77-123. doi:10.1016/0370-1573(87)90009-3

[5] P. Lobastie, M. C. Bordas, B. Tribollet and M. Boyer, "Stroboscopic Effect between Electronic and Nuclear Motion in Highly Excited Molecular Rydberg Sates," Physicsl Review Letters, Vol. 52, No. 19, 1984, pp. 16811684. doi:10.1103/PhysRevLett.52.1681

[6] J. Chevaleyre, C. Bordas, M. Boyer and P. Labastie, "Stark Multiplets in Molecular Rydberg States," Physicsl Review Letters, Vol. 57, No. 24, 1986, pp. 3027-3030. doi:10.1103/PhysRevLett.57.3027

[7] C. Bordas, P. F. Brevet, M. Boyer, J. Chevaleyre, P. Labastie and J. P. Perrot, "Electric-Field-Hinhered Vibrational Autoionization in Molecular Rydberg States," Physicsl Review Letters, Vol. 60, 1988, p. 917. doi:10.1103/PhysRevLett.60.917

[8] M. Lombardi, P. Labastie, M. C. Bordas and M. Boyer,
"Molecular Rydberg States: Clasical Chaos and Its Correspondence in Quantum Mechanics," Journal of Chemical Physics, Vol. 89, No. 6, 1988, 3479-3490. doi: $10.1063 / 1.454918$

[9] M. Lombardi and T. H. Seligman, "Universal and Nonuniversal Statistical Properties of Levels and Intensities for Chaotic Rydberg Molecules," Physical Review A, Vol. 47, No. 5, 1993, pp. 3571-3586. doi:10.1103/PhysRevA.47.3571

[10] J. J. Kay, S. L. Coy, V. S. Petrovi c' , B. M. Wong and R. W. Field, "Separation of Long-Range and Short-Range Interactions in Rydberg States of Diatomic Molecules," Journal of Chemical Physics, Vol. 128, No. 19, 2008, p. 194301. doi: $10.1063 / 1.2907858$

[11] D. Sugny, L. Bomble, T. Ribeyre, O. Dulieu and M. Desouter-Lecomte, "Rotovibrational Controlled-Not Gates Using Optimized Stimulated Raman Adiabatic Passage Techniques and Optimal Control Theory," Physical Review A, Vol. 80, 2009, ID 042325. doi:10.1103/PhysRevA.80.042325

[12] A. Ruiz, J. P. Palao and E. J. Heller, "Nearly Resonant Multidimensional Systems under a Transient Perturbative Interaction," Physical Review E, Vol. 80, 2009, ID 066606. doi:10.1103/PhysRevE.80.066606

[13] B. V. Chirikov, "A Universal Instability of Many-Dimensional Oscillator Systems," Physics Reports, Vol. 52, No. 5, 1979, pp. 263-379. doi:10.1016/0370-1573(79)90023-1

[14] É. V. Shuryak, "Nonlinear Resonances in Quantum Systems," Soviet Physics-JETP, Vol. 44, 1976, p. 1070.

[15] R. P. Parson, "Vibrational Adiabaticity and Infrared Multiphoton Dynamics," Journal of Chemical Physics, Vol. 88 , No. 6, 1987, pp. 3655-3666. doi: $10.1063 / 1.453865$

[16] P. S. Dardi and K. Gray "Classical and Quantum Mechanics Studies of HF in an Intense Laser Field," Journal 
of Chemical Physics, Vol. 77, No. 3, 1982, 1345-1353. doi:10.1063/1.443957

[17] G. P. Berman and A. R. Kolovsky, "Quantum Chaos in a Diatomic Molecule Interacting with a Resonant Field," Soviet Physics-JETP, Vol. 68, 1989, p. 898.

[18] G. P. Berman and A. R. Kolovsky, "Quantum Chaos in Interactions of Multilevel Quantum Systems with a Coherent Radiation Field," Soviet Physics-USP, Vol. 35, No. 4, 1992, p. 303.

[19] P. M. Morse, "Diatomic Molecules According to the Wave Mechanics II. Vibrational Lavels," Physical Review, Vol. 34, No. 1, 1929, pp. 57-64.

[20] G. P. Berman, E. N. Bulgakov and D. D. Holm, "Nonlinear Resonance and Dynamical Chaos in a Diatomic Molecule Driven by a Resonant RF Field," Physical Review A, Vol. 52, 1995, p. 3074. doi:10.1103/PhysRevA.52.3074

[21] L. Susskind and J. Glogower, "Quantum Mechanical Phase and Time Operator," Physics, Vol. 1, 1964, pp. 49-61.

[22] A. Lahiri, G. Ghosh and T. K. Kar, "Action-Angle Variables in Quantum Mechanics," Physical Letters A, Vol. 238, No. 4-5, 1998, 239-243. doi:10.1016/S0375-9601(97)00926-2

[23] P. Carruthers and M. M. Nieto, "Phase and Angle Variables in Quantum Mechanics," Reviews of Modern Physics, Vol. 40, No. 2, 1968, pp. 411-440.
doi:10.1103/RevModPhys.40.411

[24] G. J. Milburn, "Quantum and Classical Liouville Dynamics of the Anharmonic Oscillator," Physical Reviews A, Vol. 33, No. 1, 1980, pp. 674-685. doi:10.1103/PhysRevA.33.674

[25] M. Burton, "IC-443-The Interaction of a Supernova Remnant with a Molecular Cloud," Royal Astronomical Society, Vol. 28, 1987, pp. 269-276.

[26] R. Chevalier, "Supernova Remnants in MolecularClouds," Astrophysical Journal 511, 798 (1999). doi:10.1086/306710

[27] E. Wigner, "On the Quantum Correction for Thermodynamic Equilibrium," Physical Review, Vol. 40, No. 5, 1932, pp. 749-759. doi:10.1103/PhysRev.40.749

[28] K. Husimi, "Some Formal Properties of the Density Matrix," Journal of the Physical Society of Japan, Vol. 22, No. 4, 1940, pp. 264-314.

[29] R. J. Glauber, "Coherent and Incoherent States of the Radiation Field," Physical Review, Vol. 131, No. 6, 1963, pp. 2766-2788. doi:10.1103/PhysRev.131.2766

[30] E. C. G. Sudarshan, "Equivalence of Semiclassical and Quantum Mechanical Descriptions of Statistical Light Beams," Physical Review Letters, Vol. 10, No. 7, 1963, 277-279. 